\title{
Inhospital death is a biased measure of fatal outcome from bloodstream infection
}

This article was published in the following Dove Medical Press journal:

Clinical Epidemiology

\author{
Kevin B Laupland' \\ Kelsey Pasquill ${ }^{2}$ \\ Elizabeth C Parfitt ${ }^{\prime}$ \\ Gabrielle Dagasso' \\ Kaveri Gupta' \\ Lisa Steele ${ }^{2}$
}

'Department of Medicine, Royal Inland Hospital, Kamloops, BC, Canada; ${ }^{2}$ Department of Pathology and Laboratory Medicine, Royal Inland Hospital, Kamloops, BC, Canada
Correspondence: Kevin B Laupland Department of Medicine, Royal Inland Hospital, 3 I I Columbia Street, Kamloops, BC V2C 2TI, Canada

$\mathrm{Tel}+$ I 2503745 I I I

Email klaupland@gmail.com
Purpose: Inhospital death is commonly used as an outcome measure. However, it may be a biased measure of overall fatal outcome. The objective of this study was to evaluate inhospital death as a measure of all-cause 30-day case fatality in patients with bloodstream infection (BSI).

Patients and methods: A population-based surveillance cohort study was conducted, and patients who died in hospital within 30 days (30-day inhospital death) were compared with those who died in any location by day 30 post BSI diagnosis (30-day all-cause case fatality).

Results: A total of 1,773 residents had first incident episodes of BSI. Overall, 299 patients died for a 30-day all-cause case fatality rate of $16.9 \%$. Most $(1,587 ; 89.5 \%)$ of the patients were admitted to hospital, and ten (5.4\%) of the 186 patients not admitted to hospital died. Of the 1,587 admitted patients, 242 died for a 30-day inhospital death rate of $15.2 \%$. A further 47 patients admitted to hospital died after discharge but within 30 days of BSI diagnosis for a 30-day case fatality rate among admitted patients of $18.2 \%$. Patients who died following discharge within 30 days were older and more likely to have dementia.

Conclusion: The use of inhospital death is a biased measure of true case fatality.

Keywords: mortality, case fatality, bacteremia

\section{Introduction}

Vital status, either survival or death, is the most commonly utilized outcome measure in clinical studies. ${ }^{1-4}$ Use of all-cause case fatality at a defined time period of observation as an outcome measure has a number of benefits not limited to the fact that it is binary, clinically relevant, and is free from subjective observer interpretation bias. However, in many studies, vital status information is available only for hospitalized patients during their inpatient stay. As a result, inhospital death is commonly used as a surrogate measure of all-cause case fatality. ${ }^{5-7}$ Management of patients without hospitalization or differential lengths of hospital stay or patient deaths following transfer to another facility or the community during the period of follow-up among admitted patients may therefore introduce bias in the ascertainment of fatal outcome. This is of relevance given that there have been important shifts in the delivery of health care in recent years with emphasis on shorter duration of hospital stay and increased delivery of advanced care in the community setting.

Despite its widespread use, the validity of inhospital death as a surrogate measure of defined-day all-cause case fatality has not been well established. The objective of this study was to assess the use of inhospital death as compared to all-cause 30 -day case 
fatality rates among a population-based cohort of patients with bloodstream infections (BSIs).

\section{Patients and methods Study population}

Surveillance was conducted in the western interior region of British Columbia, Canada (2016 population, 182,422). This geographically diverse and large area encompasses the city of Kamloops (2016 population 114,429) and a number of other smaller communities. ${ }^{8}$ Within the region, there are six hospitals with tertiary care provided at the Royal Inland Hospital in Kamloops. Most patients are cared for exclusively within the regional facilities although patients who require management for major burns, acute organ transplantation, cardiac or thoracic surgery, invasive cardiology procedures, and some other highly specialized services are transferred to other facilities outside the region for at least part of their care. Surveillance included all western interior residents with incident BSI occurred between April 1, 2010, and March 31, 2017. This study was approved by the Interior Health Research Ethics Board (201314052-I).

\section{Population-based surveillance}

Active surveillance was conducted using a population-based design. ${ }^{9}$ The regional microbiology laboratory identified all incident episodes of BSI occurring among western interior residents including those occurring in both hospitals and the community. An experienced infectious disease consultant reviewed the electronic medical record of all cases and recorded clinical and outcome variables and assigned a most likely focus of infection. Comorbid illnesses were classified using the Charlson score. ${ }^{10}$ Vital status was ascertained using the electronic health record that is populated through a direct linkage between the regional enterprise master patient index and provincial vital statistics database that registers all deaths occurring anywhere within the province.

\section{Laboratory and study definitions}

The BacT/Alert 3D System (bioMerieux, Craponne, France) was used to culture blood during the study. Blood cultures were considered as a set consisting of growth of an organism within an anaerobic/aerobic pair from a single draw. In our region, usual practice is to draw two independent sets from different sites per order. We defined a BSI incident by the first isolate per patient per clinical infection episode and repeat isolation of the same organism within 30 days were deemed to represent the same episode. Patients who had more than one incident isolates of different species within a 48-hour period were classified as having polymicrobial BSI. We excluded contaminants based on a clinical review of all available laboratory, diagnostic, and clinical information. Hospital-onset BSIs were those where the first blood culture was drawn $\geq 48$ hours after hospital admission. ${ }^{11}$ Community-onset infections were those which occurred among patients not admitted or within the first 48 hours of admission to hospital and were subcategorized as either community associated or healthcare associated by applying the criteria of Friedman et al. ${ }^{12}$ Transfers of patients between hospitals were considered to represent a single continuous admission.

Inhospital death was defined as deaths within 30 days of index BSI among patients admitted to hospital and was censored at discharge; patients who were alive in hospital or discharged alive prior to day 30 were deemed to not have inhospital death. Patients who died in any location within 30 days of BSI diagnosis were deemed to have all-cause 30-day case fatality.

\section{Statistical analyses}

Data were managed and analyzed using Stata (version 15.1; StataCorp LP, College Station, TX, USA). Prior to analysis, the individual patient's data were anonymized. The primary analysis was the comparison of inhospital and all-cause 30-day case fatality rates. An a priori specified secondary analysis included comparison between all-cause case fatality and inhospital death rates at 7, 14, 21, 30, and 90 days. Categorical data were compared using Fisher's exact test. Skewed continuously distributed variables were reported as median with IQR and were compared with the Mann-Whitney test.

\section{Results}

During the 7 years of surveillance, 1,773 area residents had first incident episodes of BSI. The median age was 67.8 (IQR, 54.4-78.4) years, and 944 (53.4\%) were male. Cases included $266(15.0 \%)$ that were hospital onset, $833(47.0 \%)$ were health care associated, and $674(38.0 \%)$ were community associated. Most $(1,587 ; 89.5 \%)$ of the patients were admitted to hospital for management as shown in Figure 1. The median length of stay for admitted patients was 9 (IQR, 5-20) days, and this did not vary significantly by the study year $(P=0.06)$.

Overall, 299/1,773 patients died within 30 days of BSI at an all-cause 30 -day case fatality rate of $16.9 \%$. Ten $(5.4 \%)$ of the 186 patients not admitted to hospital died, and therefore the death rate among those admitted to hospital was $15.2 \%$ $(242 / 1,587)$. Forty-seven patients admitted to hospital died after discharge but within 30 days of BSI diagnosis for a 
30 -day case fatality rate among admitted patients $(n=1,587)$ of $18.2 \%$. The proportion of patients who died within 30 days of hospitalization either in hospital or after discharge did not vary significantly $(P=0.7)$ during the study period.

Among the cohort of admitted patients who died within 30 days of index BSI $(n=289)$, those who died following discharge were significantly older and more likely to have dementia than those who died during hospitalization within 30 days as summarized in Table 1 .
Among the 47 patients who died after hospital discharge but within 30 days of the index BSI, most deaths were not unexpected. These included a documented palliative approach in 35 (74.5\%) cases of which 15 of these were discharged to hospice. Among the other 12 (25.5\%) patients, six were discharged to nursing homes, three patients were transferred out of the region for quaternary care, one patient was likely under palliative care (based on comorbidities but not explicitly documented as such), and details were lacking on the remaining two.

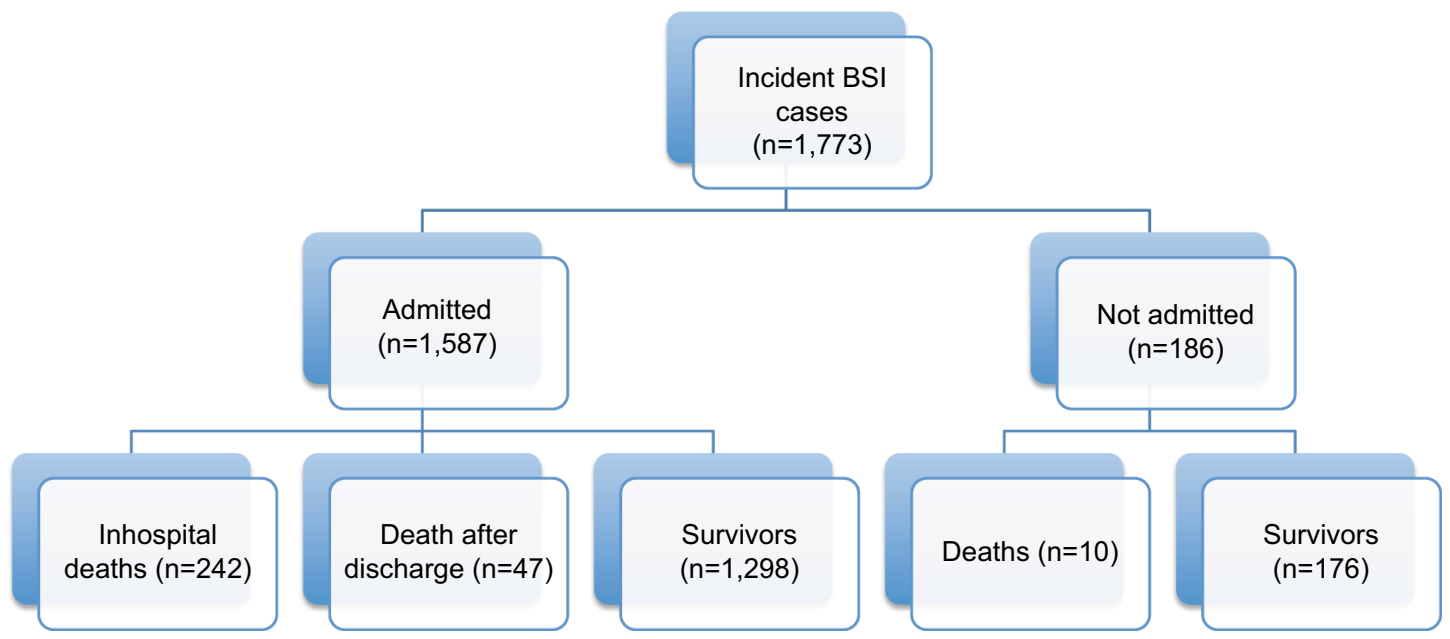

Figure I Incidence and outcomes of BSI at 30 days of follow-up, western interior of British Columbia, 20I0-20I7.

Abbreviation: $\mathrm{BSI}$, bloodstream infection.

Table I Comparison of admitted patients who died during hospitalization or after discharge within 30 days of BSI

\begin{tabular}{|c|c|c|c|c|}
\hline Variable & $\begin{array}{l}\text { Death during hospital } \\
\text { stay }(n=242)\end{array}$ & $\begin{array}{l}\text { Death after hospital } \\
\text { discharge }(n=47)\end{array}$ & $\begin{array}{l}\text { RR }(95 \% \mathrm{Cl}) \text { death } \\
\text { after discharge }\end{array}$ & $P$-value \\
\hline Median age (IQR) (years) & $72.9(63.2-81.3)$ & $78.5(64.9-84.0)$ & - & 0.035 \\
\hline Male & $128(53 \%)$ & $31(66 \%)$ & $1.2(1.0-1.6)$ & 0.1 \\
\hline \multicolumn{5}{|l|}{ Acquisition } \\
\hline Hospital onset & 79 (33\%) & II (23\%) & - & 0.6 \\
\hline Health care associated & $117(48 \%)$ & $29(62 \%)$ & & \\
\hline Community associated & $46(19 \%)$ & $7(15 \%)$ & & \\
\hline Median Charlson score (IQR) & $2(1-4)$ & $3(I-6)$ & - & 0.2 \\
\hline Myocardial infarction & $20(8 \%)$ & $5(11 \%)$ & $1.3(0.5-3.3)$ & 0.6 \\
\hline Congestive heart failure & 29 (I2\%) & $9(19 \%)$ & $1.6(0.8-3.2)$ & 0.2 \\
\hline Peripheral vascular disease & $14(6 \%)$ & $3(6 \%)$ & I.I (0.3-3.7) & 0.9 \\
\hline Cerebrovascular accident & $28(12 \%)$ & $3(6 \%)$ & $0.6(0.2-1.7)$ & 0.3 \\
\hline Hemiplegia & $3(1 \%)$ & 0 & - & 0.4 \\
\hline Chronic lung disease & $49(20 \%)$ & $10(21 \%)$ & $1.1(0.6-1.9)$ & 0.9 \\
\hline Diabetes mellitus & $44(18 \%)$ & $12(26 \%)$ & $1.4(0.8-2.5)$ & 0.2 \\
\hline Renal disease & 27 (II\%) & $7(15 \%)$ & I.I (0.5-2.4) & 0.5 \\
\hline Liver disease & $36(15 \%)$ & $5(11 \%)$ & $0.7(0.3-1.7)$ & 0.4 \\
\hline Peptic ulcer disease & $15(6 \%)$ & $2(4 \%)$ & $0.7(.2-2.9)$ & 0.6 \\
\hline Cancer & $90(37 \%)$ & $21(45 \%)$ & $1.2(0.8-1.7)$ & 0.3 \\
\hline Dementia & $20(8 \%)$ & $13(28 \%)$ & $3.3(1.8-6.2)$ & $<0.001$ \\
\hline Rheumatic disease & $14(6 \%)$ & $4(9 \%)$ & $1.5(0.5-4.3)$ & 0.5 \\
\hline HIVIAIDS & $2(1 \%)$ & 0 & & \\
\hline
\end{tabular}

Abbreviation: BSI, bloodstream infection. 
The degree to which inhospital death underestimated 30-day case fatality increased with longer durations of followup as shown in Figure 2. There was a progressive increase in the proportion of post-discharge deaths with longer followup duration that was most notable at day 90 , where $31 \%$ (120/384) deaths were post-hospital discharge (Figure 2).

\section{Discussion}

We demonstrate that, in our population, determination of the proportion of patients with BSI who die is dependent on how death is ascertained. All-cause 30-day case fatality rate was established as the "gold standard" outcome for our study. ${ }^{13}$ This choice was based on the fact that this outcome was independent of decisions to admit or discharge patients and 1 month (28 or 30 days) of follow-up has traditionally been the standard duration of follow-up in clinical trials and observational studies of sepsis and BSI. ${ }^{7}$ We observed that the "true" all-cause 30 -day case fatality rate was $16.9 \%$. If one considers all 1,773 incident BSI cases in this study including patients managed in the community and in hospital, the 242 deaths identified in hospital represent a much smaller proportion (13.6\%) overall. Notably, had we chosen a longer duration of follow-up these differences would have been more pronounced (Figure 2). Our results are not surprising as one may reasonably predict that if deaths following discharge are not measured the rate will be lower. However, this issue is rarely raised in literature critique. Our data provide empiric evidence to demonstrate that the use of hospital death alone will fail to ascertain the true mortality outcome. These data not only question mortality outcomes used in research studies but raise concern about benchmarking between institutions based on inhospital case fatality. Ideally, all-cause defined case fatality rates at a specified outcome period should be used and where reliable population-level vital statistics data is not available, at the very least, attempts should be made to include patients who are transferred to hospice or palliative care programs.

Not only does inhospital death underestimate the case fatality rate but also it biases the evaluation of the determinants of death. We hypothesized in advance that there may be differences in the clinical characteristics of patients among those dying in hospital vs those dying as outpatients within 30 days of BSI. Our observation that patients who died post discharge were older and more likely to have dementia supports this (Table 1). It is further possible that other comorbidities were truly different but that our study was underpowered to detect these. In our health system, there are limited hospice beds and it is possible that some of the inhospital deaths would have been post discharge and had there been ready access to these beds. However, we did not have data on all of our patients regarding curative or palliative treatment intent to evaluate this further. The difference among inhospital and 30-day case fatality rates in other jurisdictions will likely be dependent on the disposition of patients receiving palliative care.

Death is a discrete binary variable that is free from observer ascertainment bias. However, it is subject to bias if follow-up is incomplete or censored. We contend that many investigators and users of medical literature do not scrutinize the potential limitations of inhospital death as a measure of true defined follow-up death outcome and tend to consider these terms synonymous. Anecdotally, we rarely observe this issue as a discussion or limitation point in published studies

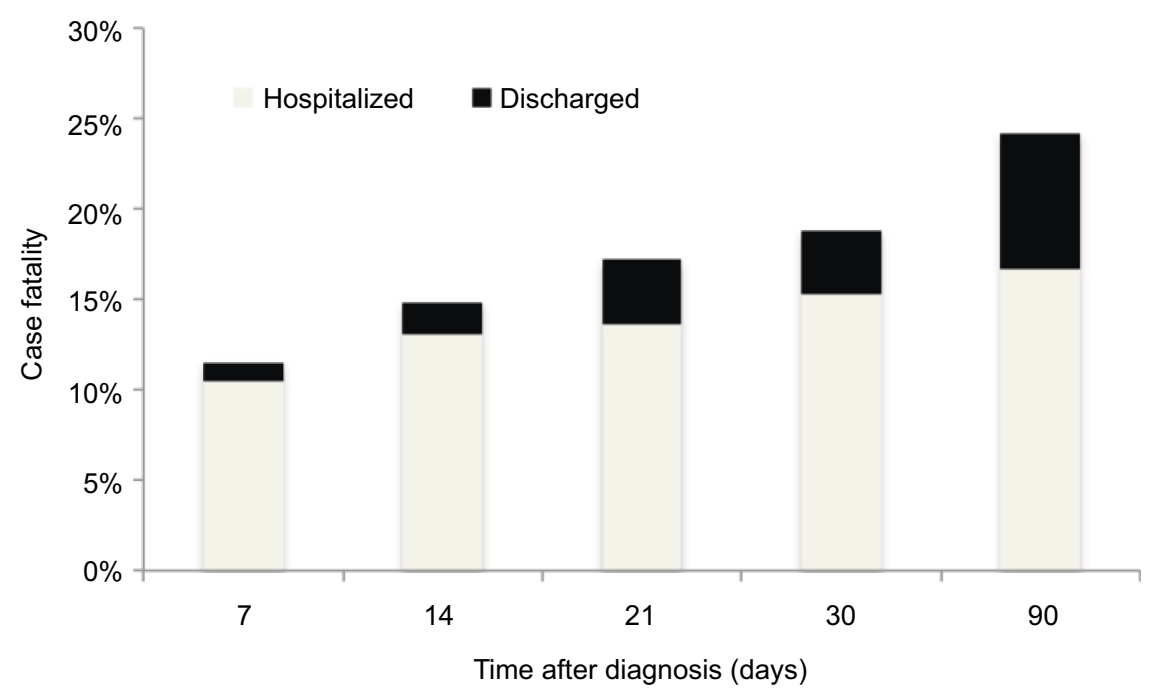

Figure 2 All-cause case fatality rates and hospital disposition at the time of death during varying periods of follow-up, western interior of British Columbia, 20I0-20I7. 
and this includes our own previous work. ${ }^{14,15}$ To derive an estimate of the use of death as outcome in contemporary studies, we searched PubMed on March 9, 2018, using terms "mortality" and "bloodstream infection" and sampled the first ten original studies that involved human subjects with a reported and evaluable death outcome. Among these, one-half only evaluated inhospital outcomes including two studies that restricted to intensive care unit (ICU) outcome alone ${ }^{16,17}$ with the other three limited to inhospital death. ${ }^{18-20}$ The other one-half of studies evaluated 30-day, ${ }^{21,22}$ 90-day, ${ }^{23,24}$ and 6-month ${ }^{25}$ all-cause deaths. We chose BSI to study this issue as a matter of convenience as we had ready access to a detailed population-based database. However, we suspect that the inhospital bias we observed is generalizable to many other conditions and jurisdictions and merits investigation elsewhere.

There are some methodological aspects of our study that merit discussion. It is a strength that we utilized a populationbased design that included all cases of incident BSI occurring among residents of a well-defined geographical area. As a result, sampling bias was minimized. ${ }^{26}$ By virtue of this design, we were able to evaluate not only the number of patients who died and who were never admitted but also those who died after hospital admission. It is a limitation of our study that the review of clinical aspects of patients was retrospective and therefore ascertainment of diagnosis, comorbidities, and treatment intent was dependent on the information existing in the medical charts. It is also an important consideration that we made no attempt to adjudicate whether the BSI was causative in a patient's death or not. Although it is probable that deaths within a short period of time following a BSI diagnosis are largely attributable to the infection, other determinants including comorbidities or downstream complications of the acute illness were likely increasingly causative for death in later stages of follow-up. In any case, attribution of cause of death to a specific disease is fraught with difficulty and the case for using all-cause death as an outcome measure has been convincingly argued..$^{13}$ It is also noteworthy that we a priori defined an admission as a continuous event including interhospital transfers as this is common between our regional hospitals. Had we only included the index hospital admission we suspect that more pronounced findings would have been observed. Finally, we relied on the information registered in the electronic database to establish vital status without further formalized verification. However, based on the fact that these data are populated from provincial vital statistics it is likely highly accurate.

\section{Conclusion}

We observe that in our population-based cohort of patients with BSI, inhospital death is a biased measure of true allcause case fatality. Not only the use of inhospital death underestimates the true death burden and this increases with the duration of follow-up, but also it introduces a bias in the evaluation of the determinants of death among study subjects. Inhospital death is commonly used as an outcome measure in published studies. Although the use of defined-day all-cause case fatality is not feasible, the limitations associated with inhospital death need to be emphasized in the interpretation of studies investigating the outcome of infectious diseases and other conditions. It is likely that the degree of bias associated with the use of inhospital death will vary among different institutions and populations, and further investigation in other jurisdictions is warranted.

\section{Acknowledgements}

This study was supported in part by the Strand Infectious Diseases Innovation Fund, Royal Inland Hospital Foundation. The sponsor had no role in the conception, conduct, or reporting of the study.

\section{Author contributions}

All the authors substantially contributed to conception and design, data acquisition, or data analysis and interpretation. All the authors contributed to the drafting of the article or critically revising it for important intellectual content. All the authors provided the final approval of the version to be published, and agreed to be accountable for all aspects of the work in ensuring that questions related to the accuracy or integrity of the work are appropriately investigated and resolved.

\section{Disclosure}

The authors report no conflicts of interest in this work.

\section{References}

1. Goto M, Al-Hasan MN. Overall burden of bloodstream infection and nosocomial bloodstream infection in North America and Europe. Clin Microbiol Infect. 2013;19(6):501-509.

2. Laupland KB. Incidence of bloodstream infection: a review of population-based studies. Clin Microbiol Infect. 2013;19(6):492-500.

3. Uslan DZ, Crane SJ, Steckelberg JM, et al. Age- and sex-associated trends in bloodstream infection: a population-based study in Olmsted County, Minnesota. Arch Intern Med. 2007;167(8):834-839.

4. Søgaard M, Nørgaard M, Dethlefsen C, Schønheyder HC. Temporal changes in the incidence and 30-day mortality associated with bacteremia in hospitalized patients from 1992 through 2006: a population-based cohort study. Clin Infect Dis. 2011;52(1):61-69. 
5. Ziegler MJ, Pellegrini DC, Safdar N. Attributable mortality of central line associated bloodstream infection: systematic review and metaanalysis. Infection. 2015;43(1):29-36.

6. Cavalcanti AB, Bozza FA, et al. Effect of a quality improvement intervention with daily round checklists, goal setting, and clinician prompting on mortality of critically ill patients: a randomized clinical trial. JAMA. 2016;315(14):1480-1490.

7. Kalil AC, van Schooneveld TC, Fey PD, Rupp ME. Association between vancomycin minimum inhibitory concentration and mortality among patients with Staphylococcus aureus bloodstream infections: a systematic review and meta-analysis. JAMA. 2014;312(15):1552-1564.

8. Interactive Health Geographies. BC statistics. Available from: http:// bcstats.gov.bc.ca/StatisticsBySubject/Geography/ReferenceMaps/ Health.aspx. Accessed November 18, 2017.

9. Vergison A, Tuerlinckx D, Verhaegen J, Malfroot A. Belgian invasive pneumococcal disease study G. epidemiologic features of invasive pneumococcal disease in Belgian children: passive surveillance is not enough. Pediatrics. 2006;118(3):e801-809.

10. Charlson ME, Pompei P, Ales KL, Mackenzie CR. A new method of classifying prognostic comorbidity in longitudinal studies: development and validation. J Chronic Dis. 1987;40(5):373-383.

11. Morin CA, Hadler JL. Population-based incidence and characteristics of community-onset Staphylococcus aureus infections with bacteremia in 4 metropolitan Connecticut areas, 1998. J Infect Dis. 2001;184(8):1029-1034.

12. Friedman ND, Kaye KS, Stout JE, et al. Health care--associated bloodstream infections in adults: a reason to change the accepted definition of community-acquired infections. Ann Intern Med. 2002;137(10): 791-797.

13. Prasad V, Lenzer J, Newman DH. Why cancer screening has never been shown to "save lives"--and what we can do about it. $B M J$. 2016;352:h6080.

14. Laupland KB, Pasquill K, Parfitt EC, Naidu P, Steele L. Burden of community-onset bloodstream infections, Western Interior, British Columbia, Canada. Epidemiol Infect. 2016;144(11):2440-2446.

15. Laupland KB, Kibsey PC, Gregson DB, Galbraith JC. Populationbased laboratory assessment of the burden of community-onset bloodstream infection in Victoria, Canada. Epidemiol Infect. 2013;141(1): 174-180.
16. Schwab F, Geffers C, Behnke M, Gastmeier P. ICU mortality following ICU-acquired primary bloodstream infections according to the type of pathogen: A prospective cohort study in 937 Germany ICUs (20062015). PLoS One. 2018;13(3):e0194210.

17. Spatenkova V, Bradac O, Fackova D, Bohunova Z, Suchomel P. Low incidence of multidrug-resistant bacteria and nosocomial infection due to a preventive multimodal nosocomial infection control: a 10-year single centre prospective cohort study in neurocritical care. BMC Neurol. 2018;18(1):23.

18. Parreco JP, Hidalgo AE, Badilla AD, Ilyas O, Rattan R. Predicting central line-associated bloodstream infections and mortality using supervised machine learning. J Crit Care. 2018;45:156-162.

19. Geng TT, Xu X, Huang M. High-dose tigecycline for the treatment of nosocomial carbapenem-resistant Klebsiella pneumoniae bloodstream infections: A retrospective cohort study. Medicine. 2018;97(8):e9961.

20. Mclaughlin MM, Miglis C, Skoglund E, Wagner J, Advincula MR, Scheetz MH. Effect of elevated imipenem/cilastatin MICs on patient outcomes in gram-negative bloodstream infections. J Glob Antimicrob Resist. 2018;261-263.

21. Espejo E, Andrés M, Borrallo RM, et al. Bacteremia associated with pressure ulcers: a prospective cohort study. Eur J Clin Microbiol Infect Dis. 2018;37(5):969-975.

22. Xie Y, Tu B, Zhang X, et al. Investigation on outcomes and bacterial distributions of liver cirrhosis patients with gram-negative bacterial bloodstream infection. Oncotarget. 2018;9(3):3980-3995.

23. Daneman N, Rishu AH, Pinto R, et al. 7 versus 14 days of antibiotic treatment for critically ill patients with bloodstream infection: a pilot randomized clinical trial. Trials. 2018;19(1):111.

24. Kronen R, Hsueh K, Lin C, Powderly WG, Spec A. Creation and assessment of a clinical predictive calculator and mortality associated with Candida krusei bloodstream infections. Open Forum Infect Dis. 2018;5(2):ofx253.

25. Venkataraman R, Divatia JV, Ramakrishnan N, et al. Multicenter observational study to evaluate epidemiology and resistance patterns of common intensive care unit-infections. Indian J Crit Care Med. 2018;22(1): 20-26.

26. Laupland KB. Defining the epidemiology of bloodstream infections: the 'gold standard' of population-based assessment. Epidemiol Infect. 2013;141(10):2149-2157.
Clinical Epidemiology

\section{Publish your work in this journal}

Clinical Epidemiology is an international, peer-reviewed, open access, online journal focusing on disease and drug epidemiology, identification of risk factors and screening procedures to develop optimal preventative initiatives and programs. Specific topics include: diagnosis, prognosis, treatment, screening, prevention, risk factor modification,

Submit your manuscript here: https://www.dovepress.com/clinical-epidemiology-journa

\section{Dovepress}

systematic reviews, risk and safety of medical interventions, epidemiology and biostatistical methods, and evaluation of guidelines, translationa medicine, health policies and economic evaluations. The manuscript management system is completely online and includes a very quick and fair peer-review system, which is all easy to use. 